\title{
Optonongenetic enhancement of activity in primary cortical neurons
}

\section{Silvia Ghirga, ${ }^{1, *}$ (i) Francesca Pagani, ${ }^{2}$ Maria Rosito, ${ }^{3}$ Silvia Di Angelantonio, ${ }^{3,4}$ Giancarlo Ruocco, ${ }^{1,3}$ and Marco LeONeTtI ${ }^{2}$}

${ }^{1}$ Department of Physics, Sapienza University, P.le A. Moro 5, Rome 00185, Italy

${ }^{2}$ Institute of Nanotechnology, CNR-NANOTEC, P.le A. Moro 5, Rome 00185, Italy

${ }^{3}$ Center for Life Nano Science CLNS @Sapienza, Istituto Italiano di Tecnologia, Viale Regina Elena 291, Rome 00161, Italy

${ }^{4}$ Department of Physiology and Pharmacology, Sapienza University, P.le A. Moro 5, Rome 00185, Italy

${ }^{*}$ Corresponding author: silvia.ghirga@gmail.com

\begin{abstract}
Received 12 December 2019; revised 21 February 2020; accepted 22 February 2020; posted 26 February 2020 (Doc. ID 385832);
\end{abstract} published 0 MONTH 0000

\begin{abstract}
It has been recently demonstrated that the exposure of naive neuronal cells to light-at the basis of optogenetic techniques and calcium imaging measurements-may alter neuronal firing. Indeed, understanding the effect of light on nongenetically modified neurons is crucial for a correct interpretation of calcium imaging and optogenetic experiments. Here we investigated the effect of continuous visible LED light exposure (490 nm, $0.18-1.3 \mathrm{~mW} / \mathrm{mm}^{2}$ ) on spontaneous activity of primary neuronal networks derived from the early postnatal mouse cortex. We demonstrated, by calcium imaging and patch clamp experiments, that illumination higher than $1.0 \mathrm{~mW} / \mathrm{mm}^{2}$ causes an enhancement of network activity in cortical cultures. We investigated the possible origin of the phenomena by blocking the transient receptor potential vanilloid 4 (TRPV4) channel, demonstrating a complex connection between this temperature-dependent channel and the measured effect. The results presented here shed light on an exogenous artifact, potentially present in all calcium imaging experiments, that should be taken into account in the analysis of fluorescence data. () 2020 Optical Society of America
\end{abstract}

https://doi.org/10.1364/JOSAA.385832

\section{INTRODUCTION}

Manipulation of neuronal activity is fundamental in order to understand neuronal circuits functioning. For two centuries, electrical stimulation has been used as the standard method to control and stimulate electrical activity in neurons $[1,2]$. The patch clamp technique is one of the most recognized tools to study electrical proprieties of a single cell or a single membrane channel $[3,4]$ but lacks the possibility to control activity of a big number of neurons simultaneously. Devices such as multielectrode array (MEA) attempt to remedy this problem. An MEA consists of multiple (tens to thousands) microscopic electrodes through which neural activity is recorded and stimulated. However, commercial chips have a limited number of electrodes and electrode size (hundreds of microns) limiting the measurements to a population response. Custom devices with an elevated number of smaller electrodes (tens of microns) are emerging, providing better spatial resolution, but analysis of the recorded data becomes more complex [5]. Besides the generally poor spatial specificity, electrical stimulation and recording suffer from electrical interference from the environment, intrinsic damage caused by direct contact with the electrodes, and the presence of high-frequency artifacts associated with the stimulation signal. To overcome these limitations, over the past decades, novel techniques based on optical stimulation and monitoring were designed [6]. Light has the advantage of being noninvasive and spatiotemporally controllable with high precision. In addition, no direct contact is necessary between the stimulating source and the tissue, thus preventing cell damage $[7,8]$. Optogenetic techniques are increasingly popular and are today playing a key role in the field of neuroscience [9-11] allowing the whole optical control of neuronal cells. This approach requires genetic manipulation of the cultures to selectively insert light-sensitive ionic channels (opsins) to neuron membranes, allowing them to be activated by light [12-14]. However, light on its own is not completely inert. Several studies report that light at different wavelengths and intensities may alter cellular physiology [15]. Since the 1960s infrared light has been known to have an effect on nerve cells $[16,17]$ and subsequently infrared neural stimulation (INS) was proposed as an alternative approach to achieve optical stimulation of neurons without the need for any genetic manipulation $[18,19]$. INS consists of short pulses of infrared light directly absorbed by water [20,21], by an exogenous fluorophore [22], or by gold nanoparticles [23]. 
Infrared techniques require delivery of high-power pulses and have to compete with strong absorption in water. In the past few years some investigations brought the attention to the effects of light in the visible spectrum on naive neurons. A recent work illustrates a significant inhibitory effect of blue and yellow LED light (470-570 $\mathrm{nm}$ range, $13 \mathrm{~mW}$ ) on the firing activity of different cell types (mitral cells and tufted neurons in the olfactory bulb and medium spiny neurons in the striatum) [24]. Other previous studies documented an excitatory effect of blue light on cortical neurons $[25,26]$. In all these works, the effect of light exposure appears to be due to an increase in bath or tissue temperature that leads to changes in resting membrane potential, spontaneous spiking, input resistance, membrane time constant, and synaptic activity in acute slices [27-29]. The present work is aimed to further investigate and characterize the effects of visible LED light on naive primary cortical neuron activity. We take advantage of a custom-made optical setup designed to perform wide field calcium imaging on a large portion of in vitro neuronal cultures. We demonstrate that a shift in light power (from $0.13 \mathrm{~mW} / \mathrm{mm}^{2}$ to $1.8 \mathrm{~mW} / \mathrm{mm}^{2}$ ) increases overall spontaneous calcium activity (29\%). Earlier studies identified one of the potential mechanisms behind optical stimulation of neurons in the activation of heat-sensitive ion channels transient receptor potential vanilloid 4 (TRPV4) [30]. Different TRPV channels activate at different temperatures. Here, we discuss that the low light level conditions (typically surrounding calcium imaging) may also cause the activation of these receptors through a nontrivial pathway that involve the excitation of the fluorophore Fluo-4. The complete characterization of this effect is out of the scope of this paper; nevertheless, we also investigated the role of the TRPV4 (sensitive to the temperature changes [31-33] and the responsibility for neural activation in the framework of the INS technique [21]) in the measured effect, which has been addressed elsewhere. We demonstrate that by blocking the channel, the activity enhancement effect disappears. Characterizing the effect of visible light on neurons is crucial for a correct interpretation of calcium imaging and optogenetics results. Furthermore, our approach opens the way to a novel stimulation technique of neuronal activity resorting to visible light only, avoiding genetic manipulation of the culture and the use of high-power infrared laser light. However, further investigations are necessary to better elucidate the action of light at cellular and cognitive levels.

\section{METHODS}

\section{A. Animals}

Procedures using laboratory animals were in accordance with the Italian and European guidelines and were approved by the Italian Ministry of Health (n. 253/2016-PR) in accordance with the guidelines on the ethical use of animals from the European Communities Council Directive of September 20, 2010 (2010/63/UE). All efforts were made to minimize suffering and number of animals used.

\section{B. Primary Cortical Neurons from Postnatal P0-P2 C57BL6 Mice}

Primary neuronal cultures were prepared from the brain of 0-2-d-old C57BL/6 mice using culturing protocols previously described [34]. In brief, after removal of the meninges from the whole brain, cerebral cortices of both hemispheres were isolated and digested in $0.125 \%$ trypsin for $30 \mathrm{~min}$ at $37^{\circ} \mathrm{C}$ followed by $5 \mathrm{~min}$ DNase incubation $0.3 \mathrm{mg} / \mathrm{mL}$ at RT. Cells were mechanically dissociated with a fire-polished Pasteur pipette and plated at a density of $2.5 \times 10^{5}$ on poly-L-lysinecoated glass coverslips. Neurons maintained in serum-free Neurobasal supplemented with 2\% B27, 1\% L-Glutamine and $1 \%$ Penicillin-Streptomycin solution. Cells were cultured in a controlled environment for 14 days in vitro (DIV), with a humidified atmosphere containing $5 \% \mathrm{CO}_{2}$ at $37^{\circ} \mathrm{C}$. Two days after plating cytosine arabinoside ( $\mathrm{araC}$ ) were added at a final concentration of $1.5 \mu \mathrm{M}$ to limit the proliferation of dividing nonneuronal cells. With this method we obtained $80-90 \%$ neurons, $8-15 \%$ astrocytes, and $2-5 \%$ microglia, as determined with $\beta$-tubulin III, glial fibrillary acidic protein (GFAP), and isolectin IB4 staining. Some of the experiments were carried out after the addition of 4-Aminopyridine (4-AP, $2 \mathrm{mM}, 275875$, Sigma-Aldrich), which blocks voltage-activated $K^{+}$channels. 4-AP is a potent convulsant, generally used to cause epileptiform activity in in vitro preparations [35]. Here we used 4-AP in order to increase calcium events frequency in culture with poor spontaneous activity. The experiments were repeated, with and without 4-AP, after the addition of $5 \mu \mathrm{M}$ concentration of RN1734 (R0658, Sigma-Aldrich), a selective antagonist of thermosensitive transient receptor potential vanilloid channel TRPV4 [31-33]. Additional experiments were done following the blockage of electrical activity through $1 \mu \mathrm{M}$ tetrodotoxin (TTX, L8288, Sigma-Aldrich) administration. The latter is a neurotoxin that inhibits the firing of action potentials by binding the voltage-gated sodium channels on cell membranes and blocking the passage of sodium ions into the neuron [36,37].

\section{Experimental Setup}

\section{Calcium Imaging}

Optical setup consisted of a custom-made fluorescence microscope optimized to perform wide field calcium imaging [Fig. 1(a)]. The LED light source centered at $490 \mathrm{~nm}$ (M490L3, ThorLabs) was focused on the sample loaded with Fluo-4 and excited the fluorescent molecules bound to calcium ions. The emission was selected with a fluorescence filter $(520 \mathrm{~nm}$ FB520-10, Thorlabs), collected by the objective (High-Res $5 \mathrm{X}$ 28-20-44, Optem) and displayed on the computer monitor through a CMOS camera (ORCA-Flash4.0 V2, Hamamatsu). Images were acquired with a pixel size of $1.8 \mu \mathrm{m}$ on a field of view of $2 \times 2 \mathrm{~mm}^{2}$, allowing the behavior of collective dynamics to be monitored. We synchronized the LED light and camera recording with a data acquisition (DAQ) board controlled by a Matlab code. We used a heater controller (TC-324B, Warner Instruments) to maintain a constant $32^{\circ} \mathrm{C}$ temperature in the sample incubator. The variability of the temperature during illumination was below the precision of the instrument $\left(0.1^{\circ} \mathrm{C}\right)$. 
(a)

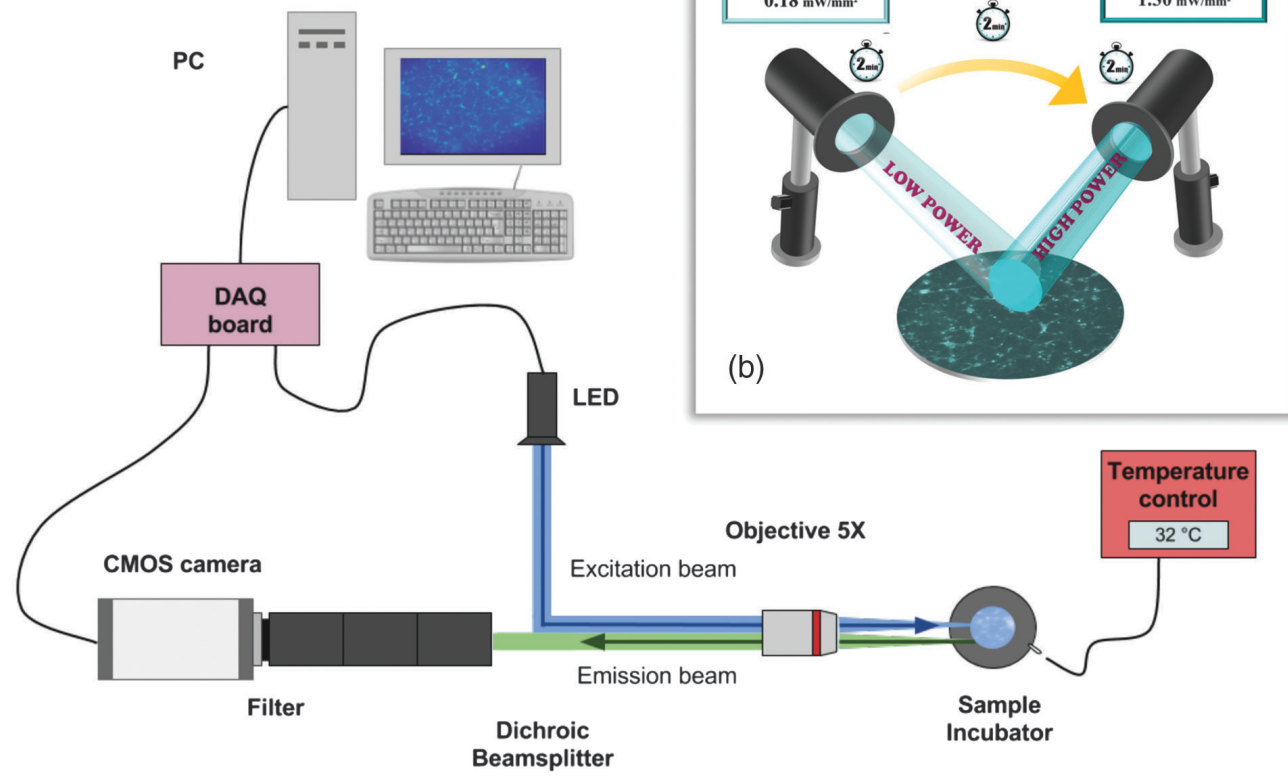

Fig. 1. Experimental setup and procedure. (a) Optical setup: LED light source focused on the sample excited Fluo-4 molecules. The emission was collected by the $5 \mathrm{X}$ objective and imaged on the display of the computer through a CMOS camera. (b) Procedure: two calcium imaging measurements were performed on the same area of the sample at low $\left(0.18 \pm 0.09 \mathrm{~mW} / \mathrm{mm}^{2}\right)$ and high $\left(1.3 \pm 0.08 \mathrm{~mW} / \mathrm{mm}^{2}\right)$ LED power illumination.

\section{Patch Clamp}

An electrophysiological recording chamber was placed under an upright microscope (Olympus BX51WI) equipped with a 14 bit CCD camera system (Cool SNAP Myo, Photometrics). Fluorescence was achieved using a Cairn Research-OptoScan monochromator and visualized with a $40 \mathrm{x}$ water-immersion objective. The system was driven by Metafluor (Molecular Devices, Foster City, CA, USA). Membrane currents were recorded with the patch-clamp amplifier (Multiclamp 700B; Molecular Devices) and acquired with Clampex 10 software (Molecular Devices).

\section{Experimental Procedure}

\section{Calcium Imaging}

Measurements of neuronal activity were carried out at DIV 7-14. Prior to each experiment, the culture was incubated for $30 \mathrm{~min}$ in a recording solution with $5 \mu \mathrm{M}$ concentration of the calcium-sensitive dye Fluo-4-AM (ThermoFisher). The culture was then washed off Fluo- 4 and placed on an observation chamber. All experiments were performed at $32^{\circ} \mathrm{C}$. Calcium imaging measurements were done under two experimental conditions [Fig. 1(b)]: at first data were recorded using lowpower light for excitation $\left(0.18 \mathrm{~mW} / \mathrm{mm}^{2}\right)$, and after $2 \mathrm{~min}$. a second measurement was performed on the same area of the sample using high-power illumination $\left(1.3 \mathrm{~mW} / \mathrm{mm}^{2}\right)$. A third measurement at low-power light was done to check the reversibility of the phenomenon. Light power at the sample plane was directly measured with an optical power meter and was reduced compared to LED output light due to the absorption by optical components in the path (filter and beamsplitter). Spontaneous activity was recorded as image sequences of 150-300 s in duration, with an acquisition speed of $1 \mathrm{~Hz}$. A low acquisition speed was required to have enough signal-to-noise ratio in low-power measurements.

\section{Patch Clamp}

Fluo-4 loaded neurons were placed in the recording chambers replacing a culture medium with a normal external solution (NES) containing (in $\mathrm{mM}$ ) $140 \mathrm{NaCl}, 2.5 \mathrm{KCl}, 2 \mathrm{CaCl}_{2}$, $2 \mathrm{MgCl}_{2}, 10$ HEPES-NaOH, and 10 glucose $(\mathrm{pH}=7.3$ with $\mathrm{NaOH}$; osmolarity $300 \mathrm{mOsm}$ ). Patch clamp recordings were obtained in a whole-cell configuration, using borosilicate glass electrodes (4-5 M) filled with an intracellular solution containing (in mM) $140 \mathrm{KCl}, 10 \mathrm{HEPES}, 2 \mathrm{Mg}$-ATP, $0.01 \mathrm{CaCl}_{2}$, $2 \mathrm{MgCl}_{2}$, and 0.5 EGTA $(\mathrm{pH}=7.3$ with $\mathrm{KOH}$; osmolarity 290 $\mathrm{mOsm}$ ). Neurons were voltage clamped at $-70 \mathrm{mV}$ for sEPSC recordings. Currents were filtered at $2 \mathrm{KHz}$, digitized $(10 \mathrm{KHz})$, and acquired with Clampex 10 software (Molecular Devices). Recording on the high-frequency channel ensures avoiding the voltage deflection artifact, previously reported for blue light in electrodes [38]. Neuronal activity was recorded in the presence of fluorescent light $\left(1.4 \mathrm{~mW} / \mathrm{mm}^{2} \pm 0.08\right.$; wavelength $488 \mathrm{~nm}$; exposure time 100-150 ms) for 10-12 min, after their sEPSCs (excitatory postsynaptic currents) were monitored for $5 \mathrm{~min}$ in the absence of fluorescence. In control experiments, cells were recorded for $15 \mathrm{~min}$ in the absence of fluorescent light. All recordings were performed at $32^{\circ} \mathrm{C}$. 


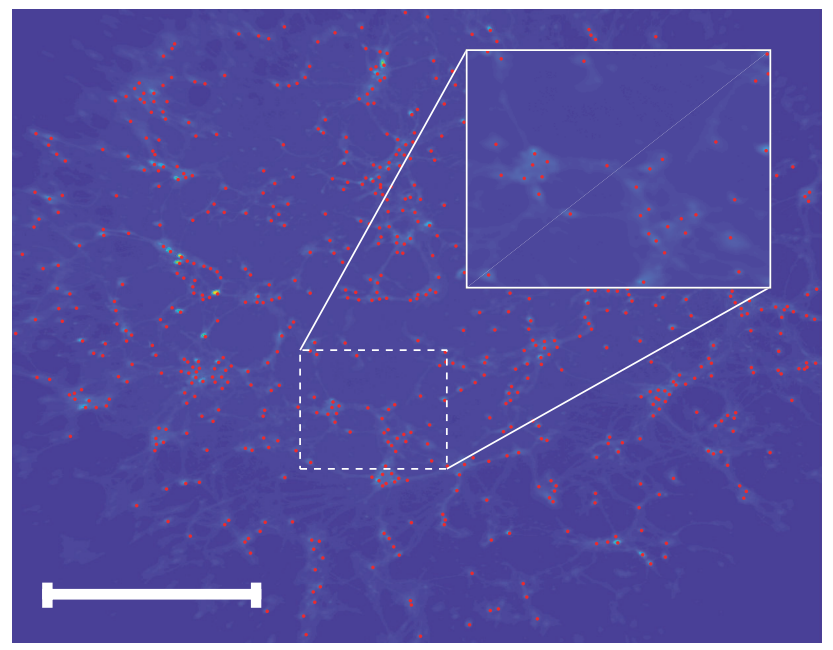

Fig. 2. Neuron detection. Data were analyzed in the frequency domain via two-dimensional Fourier transform and suitably filtered to eliminate high-frequency noisy components; neurons position were identified as the local maxima of the matrix. Scale bar: $500 \mu \mathrm{m}$.

\section{E. Data Analysis}

\section{Calcium Imaging}

For each area and each value of the power intensity, data were acquired and saved as three-dimensional matrix, a sequence of $1600 \times 1600$ pixels images. Extracting information from data required two steps: neuron recognition and calcium event detection.

On each area, neurons positions were identified by analyzing the mean frame over time of the high-power recording. This matrix was evaluated in the frequency domain via twodimensional Fourier transform and suitably filtered to eliminate high-frequency noisy components; then the local maxima of the matrix are selected. For each of these points, mean intensity mediated on a $10 \times 10$ pixel $\left(\approx 25 \times 25 \mu \mathrm{m}^{2}\right)$ area around them was calculated, and the most intense were selected and identified as cell centroids Fig. 2. We selected the most intense cells in order to reduce the signal-to-noise ratio (SNR), especially in noisy low-power measurements. To assess that this choice did not affect the interpretation of the results, we carefully compared cells with a different degree of dye load, and we observed no correlation with their activity.

Once the cell positions (500-800 for each area scanned) had been retrieved, their fluorescence intensity traces as a function of time were collected, both in low- and high-power conditions. Intensity traces were filtered using a previously described [39] modified Perona-Malik filter, an edge-enhancing denoising algorithm that performs smoothing within slow varying regions and prevents smoothing across fast varying regions (preserving fast varying fluctuation events associated with the electrophysiological activity of the neurons). On the filtered traces, a putative event is detected when a series of conditions are satisfied: at the onset the fluorescence intensity and the slope of the trace show an increase; at the offset the slope of the trace decreases, and a certain time interval occurs within the onset and the offset [39]. Each putative transient was then fitted in a two-step procedure with a model function composed of a single-exponential rise and a double exponential decay to obtain the amplitude of the signal, and the time constant of rise and decay. We selected calcium signals when the amplitude of the fitted transient exceeded $7 \%$ of the baseline value [Figs. 3(a) and 5(a)]. The threshold for peak detection was chosen as 2 s.d. of the fluorescence trace at rest from low-power measurement $(3.5 \pm 0.3 \%$ relative fluorescence change $\Delta F / F, n=30)$ and is in accordance with the average somatic $\Delta F / F$ transients corresponding to a single action potential event $(7.2 \% \pm 0.2)$, previously described through simultaneous optical and electrical recordings [40].

We recognized and discarded nonneuronal signals by analyzing calcium transient dynamics. Astrocyte calcium transients are characterized by a slowest rise time compared to neuronal signals; thus, we selected only neuronal signals by discarding calcium transients with a rise time higher than $3 \mathrm{~s}$.

In the total $4 \mathrm{~min}$ recording ( $2 \mathrm{~min}$ low power, $2 \mathrm{~min}$ high) from 60 to 150 spontaneously active neurons were found for each glass. To determine the effect of light power, the number of calcium events in low-power condition and high-power condition was statistically compared, through a paired unilateral Wilcoxon rank sum test. We obtained a certain number " $n$ " of glass in which light power significantly increases the number of calcium events. To establish if these positive results are due exclusively to a repetition of type I error and thus the null hypothesis ("light power increases do not augment number of calcium events") is true, we calculated the probability to get by chance " $n$ " statistically significant results over $\mathrm{N}$ total glass measured, as

$$
p=\sum_{m=n}^{N}\left(\begin{array}{c}
m \\
N
\end{array}\right) \alpha^{m}(1-\alpha)^{N-m},
$$

where $\alpha$ is the probability that the test failed rejecting the null hypothesis $(0.05)$. The relative increase was calculated for each neuron and the average increase was calculated as

$$
\left\langle\frac{\text { Events }_{H}-\text { Events }_{L}}{\text { Events }_{L}}\right\rangle \text {. }
$$

We performed a populations analysis computing the mean number of events, the average events shape, and network synchronization on each slide showing significant increment of neuron-by-neuron activity. We compared values obtained at low and high power by Wilcoxon rank sum tests and the ANOVA test, including low/high light as factors. Synchronicity of the network was evaluated as the relative number of simultaneous neural events.

\section{Patch Clamp}

sEPSCs were identified based on a template created for each neuron using 30 to 50 single events for each trace. All events recognized through the template search function were visualized, identified, and accepted by manual analysis. Data were analyzed offline with Clampfit 10 software; Origin 7 software was used for statistical analysis of the electrophysiological recording. 
(a)

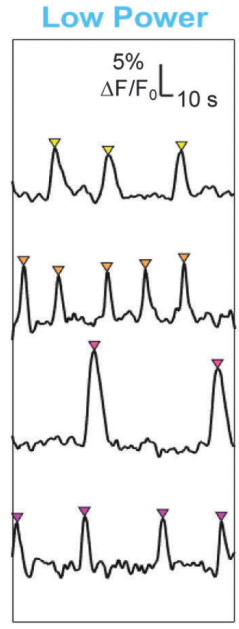

High Power

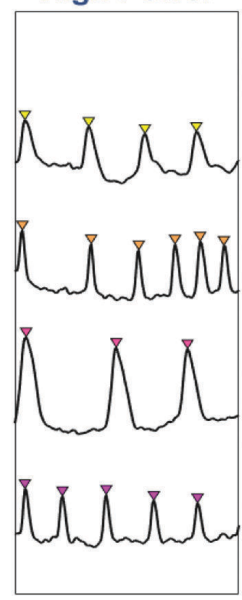

(b)

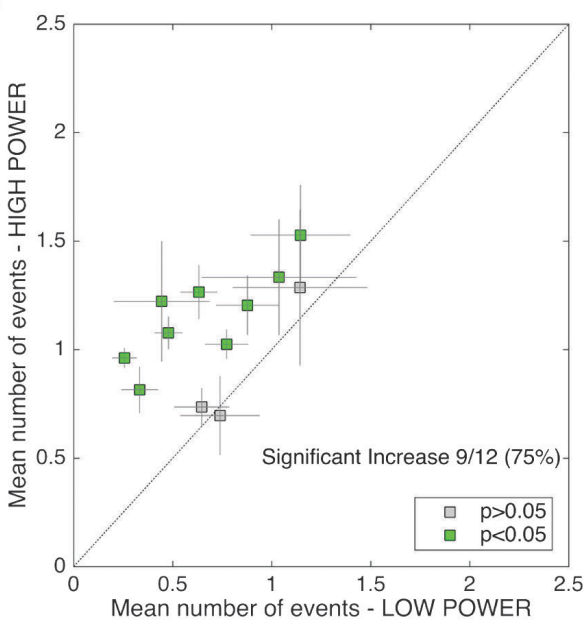

(c)

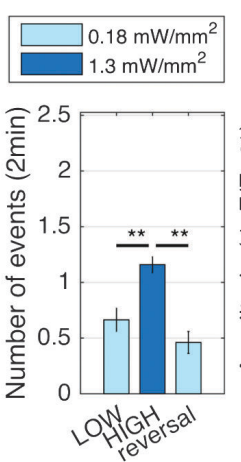

(d)

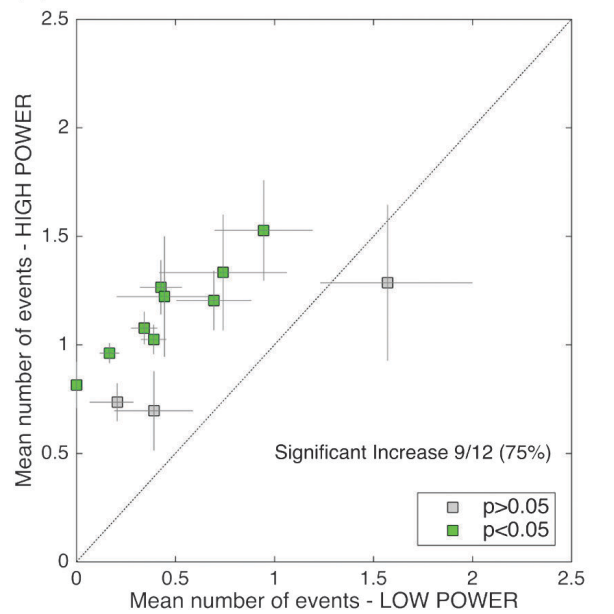

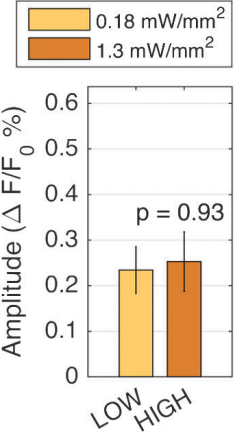
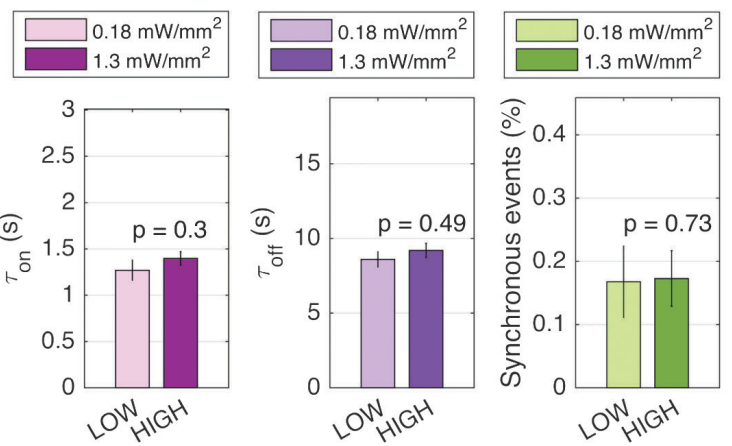

(e)

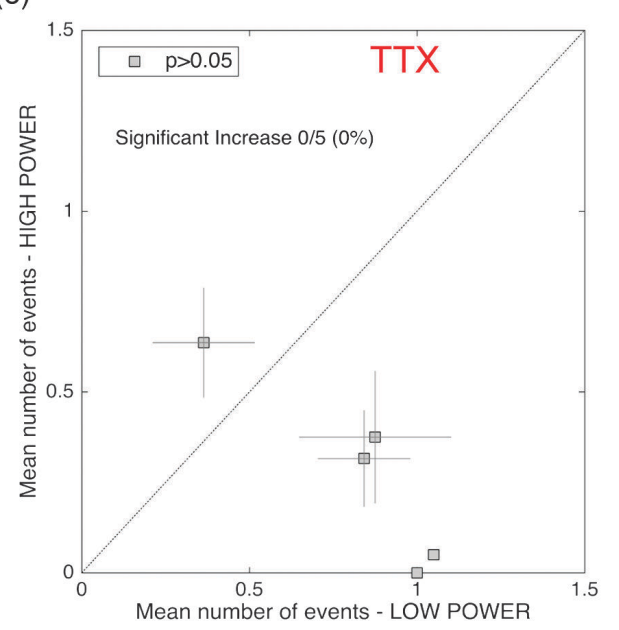

Fig. 3. (a) Fluorescence intensities traces of individual neurons, representative of the most active cells, filtered with a modified Perona-Malik filter from low (left) and high (right) light power measurements. (b) Effect produced by increasing LED power on 12 slides $\left(p=3.7 * 10^{-10}\right)$. See Data File 1 for underlying values. (c) Populations analysis: mean number of events, peaks amplitude, rise time constant, decay time constant, and synchronization, in low- and high-power conditions. Rank sum test, $N=9$. (d) Number of calcium events came back to control values when cultures are exposed again to low-power light after high-power stimulation. See Data File 1 for underlying values. (e) Results obtained by increasing LED power on five slides treated with TTX.

\section{RESULTS}

We exploited a wide field high-resolution calcium imaging setup [Fig. 1(a)] to study the influence of LED illumination on spontaneous calcium activity of primary cortical neurons from early postnatal mice. By DIV 7 neuronal cortical cultures formed an active network and showed spontaneous activity. We recorded spontaneous calcium transients on $12 \mathrm{~mm}$ glasses at DIV 7-14, loading cells with Fluo-4 AM. For each slide we scanned several large areas $\left(2 \times 2 \mathrm{~mm}^{2}\right)$ and for each area we performed two measurements: one using low-power LED light $(490 \mathrm{~nm}$ 
wavelength, $120 \mathrm{~s}, 0.18 \mathrm{~mW} / \mathrm{mm}^{2}$ ) for excitation, the other using higher LED power illumination [490 $\mathrm{nm}$ wavelength, $120 \mathrm{~s}, 1.3 \mathrm{~mW} / \mathrm{mm}^{2}$ ) Fig. 1(b)]. Data from a 2 min recording were collected and analyzed through a custom-made algorithm designed to recognize cells (Fig. 2), select neuronal traces, and sort calcium events, discarding events characterized by the slow rise time typical of astrocytes (see methods). We analyzed 65 areas from $N=12$ slides from five different cultures. For each slide from 60 to 150 spontaneously active neurons were detected, and the number of calcium events per neurons at low and high power were statistically compared (paired unilateral Wilcoxon rank sum test) to evaluate the effect of light power. In $n=9$ out of $N=12(75 \%)$ slides scanned, light induced a significant $(p<0.05)$ enhancement of activity, namely an increase in number of calcium events per neurons as shown in Figs. 3(a) and 3(b). The validity of these results is assessed by the probability to obtain by chance " $n$ " significant events over $\mathrm{N}$ experiments, i. e. the probability to repeat type I error $n$ time, which is $p=3.7 * 10^{-10}$. The average relative increase per neuron was $29 \pm 6 \%$. The phenomenon was reversible, as the number of calcium events came back to control values on $75 \%$ of slides when cultures were then exposed to the low-power light stimulation $\left(p=3.7 * 10^{-10}\right)$ Fig. $3(\mathrm{~d})$. Populations analysis was done by selecting statistically significant slides and comparing the average proprieties (number of events, amplitude and time constants) from low- and high-power measurements. We observed that relative high-power illumination induces a significant increase in the mean number of events $(N=9$, $p=0.0014$, rank sum test; $p=0.001, F=15$, ANOVA test) without affecting the event amplitude, and rise and decay time constants ( $p>0.05, N=9$, rank sum test), as shown in figure Fig. 3(c). Notably, the increase in network activity did not affect the network synchronization ( $p>0.05, N=9$, rank sum test), measured as the relative number of simultaneous events [Fig. 3(c)].

Parallel patch clamp recordings of spontaneous network activity on primary cortical neurons loaded with Fluo-4 AM confirmed that the switch on of a fluorescent light source (488 nm, $1.4 \pm 0.07 \mathrm{~mW} / \mathrm{mm}^{2}$, fluorescent light exposure: $100-150$ every second) significantly increases synaptic current frequency by $86 \%\left(n=6 ;{ }^{*} p<0.05\right.$, paired t-test $)$, as shown in Fig. 4(b), without affecting sEPSC amplitude $(9.2 \pm 2.8 \mathrm{pA}$ control; $8.2 \pm 1.2 \mathrm{pA}, 488 \mathrm{~nm}$ light on; $n=5 ; p>0.1$, paired t-test; data not shown), suggesting an increase in the release of neurotransmitters. The increase in sEPSC frequency was not simply due to the switch on of the $488 \mathrm{~nm}$ fluorescent light; indeed, when the experiments were performed either on Fluo4 AM loaded cells without light switch on, or in unloaded cells exposed to the same light stimulation, the sEPSC frequency remained unaltered [ $p>0.4, n=6$ paired t-test; Fig. 4c)].

To investigate the mechanisms involved in the light-induced effect observed, we repeated the calcium imaging experimental protocol on cultures under different conditions: treated with 4-Aminopyridine (4-AP, $2 \mathrm{mM}$ ), a $K^{+}$channel blocker; treated with 4-AP and loaded with the fluorophore Oregon Green BAPTA-1 (OGB, $5 \mu \mathrm{M})$; in the presence of tetrodotoxin (TTX, $1 \mu \mathrm{M}$ ), a selective blocker of voltage-activated sodium channels; in calcium free extra-cellular solution (0 calcium, $1 \mathrm{mM}$ EGTA); in the presence of RN1734 $(5 \mu \mathrm{M})$, a selective antagonist of thermosensitive TRPV4 in the copresence of 4-AP and RN1734.

Cultures treated with 4-AP showed enhanced spontaneous and synchronous activity compared to control, but the effect of light power on these cultures gave similar results: the increase in light power caused a significant increment of calcium events on $75 \%$ of the slides (rank sum $p=4 * 10^{-7}, N=8$ slides, from four cultures, 42 total area, 1000 neurons), as shown in Fig. 5(b). The average increase per neuron was $58 \pm 3 \%$, almost twice the average increment in the absence of 4-AP. Population analysis results are reported in Fig. 5(c) $(N=6, p=0.046$, rank sum test; $p=0.06, F=4.13$, ANOVA test).

In order to assess if the effect could be generalized to other fluorescent calcium indicator, a subsample of the cultures treated with 4-AP was loaded with OGB instead of Fluo-4.
360

361

362

363

364

365

366

367

368

369

370

371

372

373

374

375

376

377

378

379

380

381

382

383

384

385

386

387

388

389

390

391

392

393

394

395

396 (a)

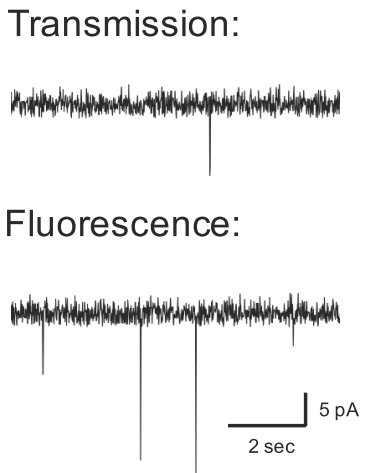

(b)

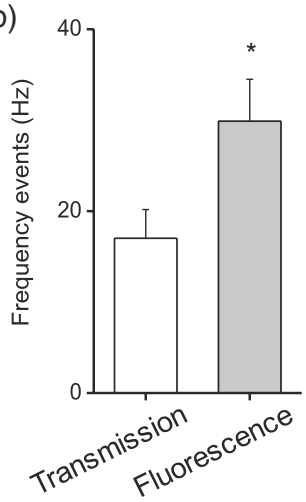

(c)

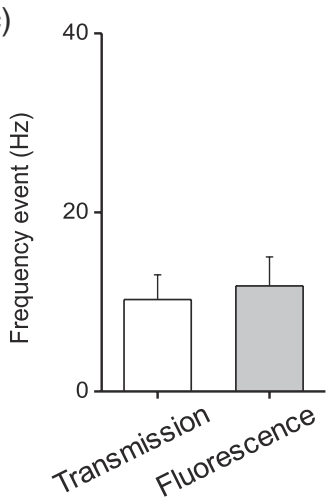

(d)

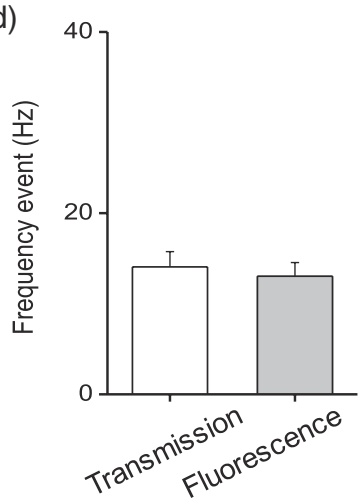

Fig. 4. (a) Representative traces of spontaneous neuronal activity of whole-cell recordings from Fluo-4 loaded primary cortical neurons, under transmission illumination (upper trace) and under exposure to fluorescence illumination (488 wavelength, low-power light; lower trace). (b) Bar graph of corresponding mean frequency event measured before (white bar) and after (gray bar) exposure of cells to fluorescence illumination $(n=6$; $*, p<0.05$, paired t-test). (c) Mean frequency event measured in unloaded primary cortical culture by patch clamp recordings, before (white bar) and after (gray bar) exposure of cells $(n=4 ; p>0.1$, paired t-test) to fluorescence illumination ( 488 wavelength, low-power light). (d) Mean frequency event measured in Fluo-4 loaded primary cortical culture treated with RN1734 (5 mM, TRPV4 blocker). Patch clamp recordings showed that in presence of the TRPV4 blocker, interevent interval of spontaneous synaptic events was not increased by the exposure to the fluorescent light (transmission: $77.9 \pm 11.9 \mathrm{~ms}$; fluorescence light: $82.7 \pm 10.6 \mathrm{~ms} ; n=6 ; p>0.4$, paired t-test). 
(a)

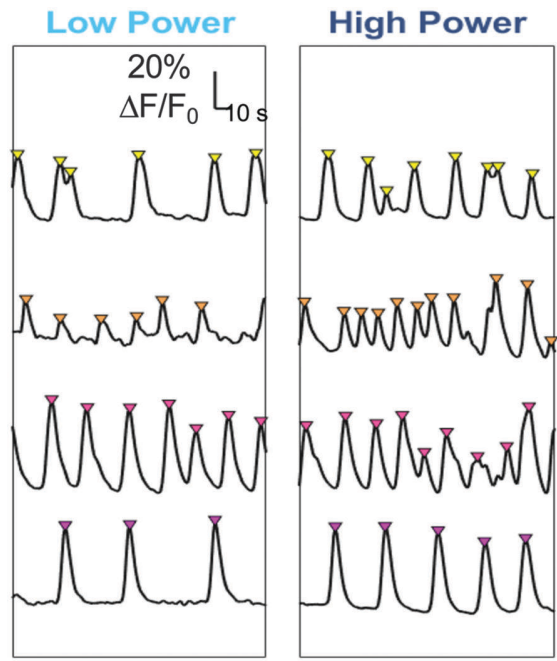

\section{4-AP treated cultures}

(b)

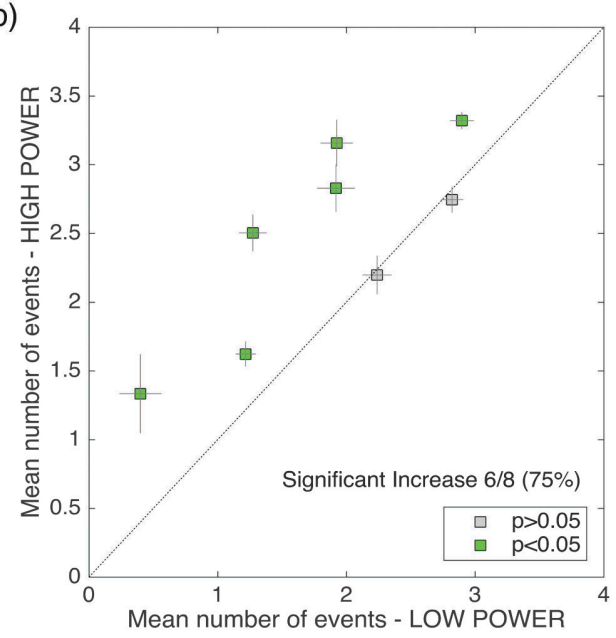

(c)
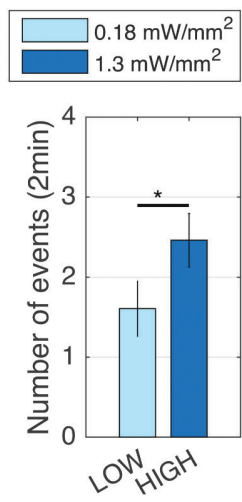
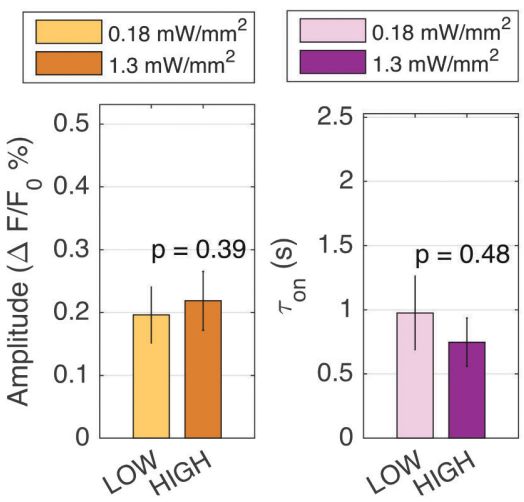
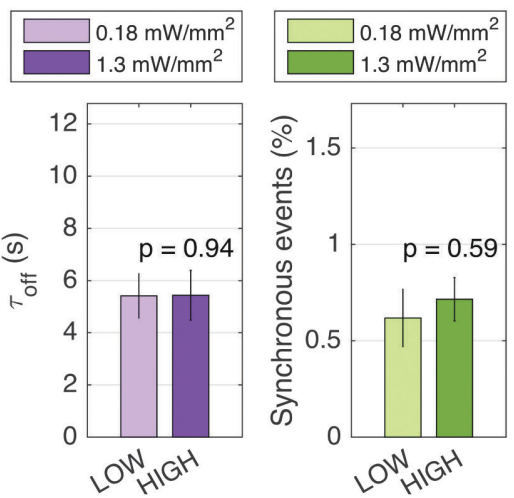

Fig. 5. (a) Fluorescence intensity traces of individual neurons, representative of the most active cells, filtered with a modified Perona-Malik filter from low (left) and high (right) light power measurements in cultures treated with 4-AP. (b) Effect produced by increasing LED power on eight slides treated with 4-AP $p=4 * 10^{-7}$. See Data File 1 for underlying values. (c) Population analysis in cultures treated with 4-AP: mean number of events, peaks amplitude, rise time constant, decay time constant, and synchronization, in low- and high-power conditions. Rank sum test, $N=6$.

Oregon Green Bapta, 4-AP treated cultures

(a)

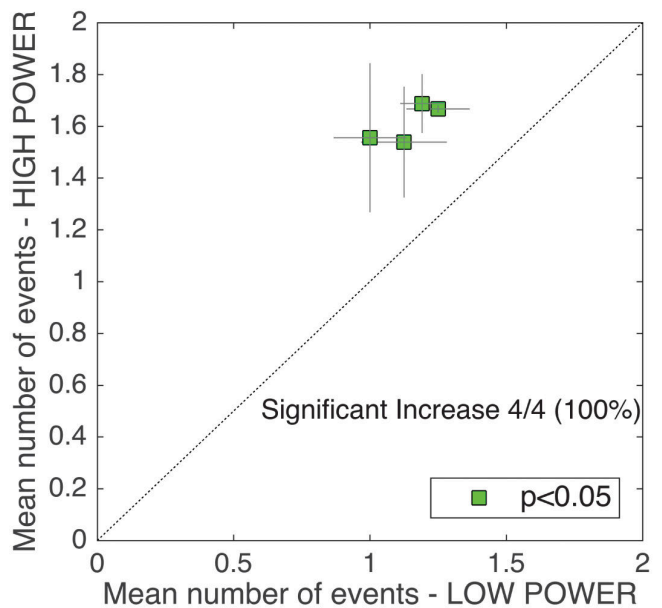

(b)
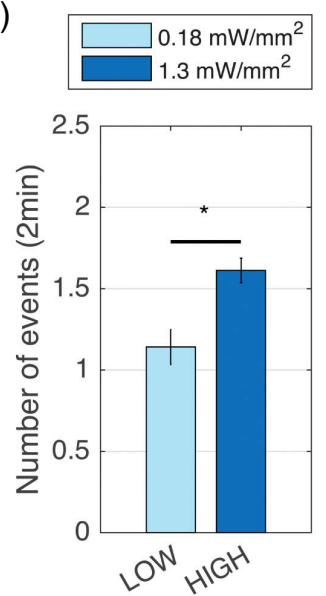

Fig. 6. Additional experiments using Oregon Green 488 BAPTA-1 (Thermofisher Scientific) as calcium indicator in 4-AP treated cultures. (a) Effect produced by increasing LED power on four slides. See Data File 1 for underlying values. (b) Population analysis: the enhancement of activity due to high-power illumination was observed also in this condition ( $p=0.014$, rank sum test). 

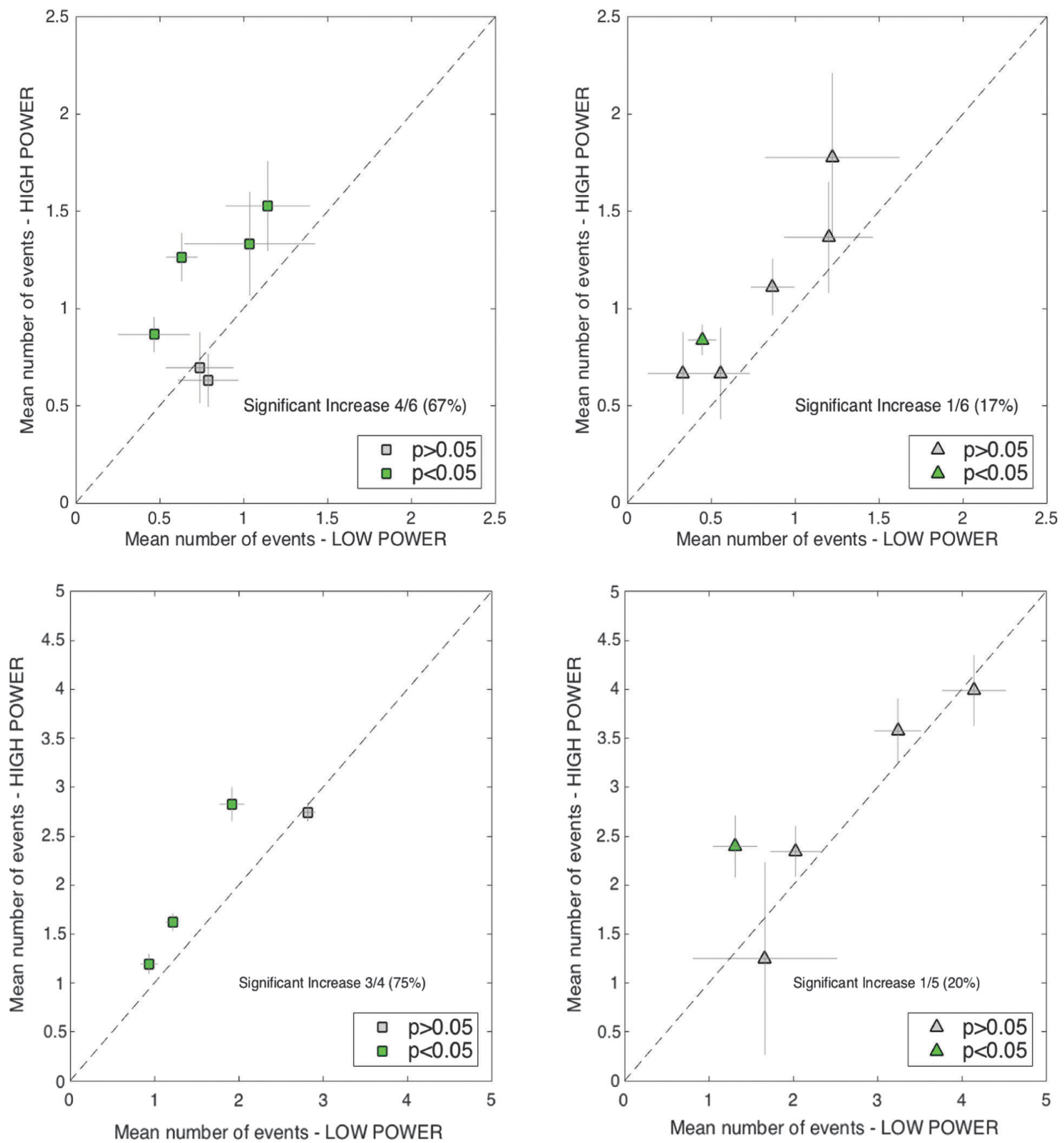

Fig. 7. Effect produced by increasing LED power in various conditions: control (top left), with 4-AP (bottom left), with RN1734 (top right) and with both 4-AP and RN1734 (bottom right). Data from control measurements (Rank sum test, $p=8.6 * 10^{-5}, p=4.8 * 10^{-4}$ ) were compared with data recorded under blockage of TRPV 4 channels (rank sum test, $p=0.23$ ). See Data File 1 for underlying values.

Because of the lower efficiency of this probe, compared to Fluo-4, when excited with blue LED light, only four samples were measured and analyzed. The weak intensity in low-power measurements made data analysis too complex to be extended to a large dataset. Results, reported in Fig. 6, showed that the enhancement of activity was observed also in this condition $(N=4, p=0.014$, rank sum test; $F=25, p=0.024$, ANOVA test).

Administration of TTX allows detection of spontaneous calcium oscillations in the absence of the spike-driven calcium entry. By inhibiting action potential network activation, the application of high-power light failed to enhance network activity (five slides, from one culture, 40 neurons), as shown in Fig. 3(e).

To disclose the source of calcium rise involved in the observed phenomenon, an additional time-lapse recording was performed in neuronal cultures maintained in a calcium-free medium. As expected, we recorded a very low calcium activity, and the light-induced effect was undetectable (six slides, from two cultures; data not shown). These data suggest that the observed enhancement of the calcium transient frequency required calcium entry from extracellular space, rather than calcium release from internal stores.

Multiple mechanisms may underlie the calcium influx from the extracellular space. Previous studies ascribe the effect of light 
exposure on neuronal activity to an increase in bath temperature. The importance of temperature for neural physiology is known: changing bath temperatures leads to changes in the functional state of neurons [27-29]. In particular it has been previously shown that, upon light stimulation of neuronal cultures, thermosensitive TRPV channels are activated [30], inducing calcium entry.

These effects can be imputed to the sensitivity of these channel-to-temperature gradients across the cell membrane. Different TRPV channels indeed have been described to be temperature-sensitive channels over a wide range of temperature gradients. In particular, TRPV4 has been shown to respond to small variations in the temperature range $\left(25^{\circ} \mathrm{C}\right.$ to $\left.40^{\circ} \mathrm{C}\right)$ typical of our experiments $\left(32^{\circ} \mathrm{C}\right)$ [31-33]. To highlight the role of these channels in the observed phenomenon, calcium transients were recorded in the presence of RN1734, a selective antagonist of TRPV4 [41]. Data reported in Fig. 7(a) show that the high-power-induced effect was prevented in cultures treated with RN1734 ( $p=0.23$, six slides, from five cultures, 21 total area, 40 neurons) and in cultures treated with both RN1734 and 4AP ( $p=0.23$, five slides, from three cultures, 17 total area, 175 neurons). Population analysis, in low- and highpower conditions, support the results $(p=0.14$, rank sum; $p=0.25, F=1.59$, ANOVA test; 4AP treated: $p=0.42$, rank sum; $p=0.75, F=0.1$, ANOVA test). In addition, whole-cell patch clamp experiments confirmed that in the presence of RN1734 the increase in spontaneous network activity frequency was abolished [Fig. 7(b)]. This ensemble of results confirms that the activation of TRPV 4 channels is responsible for the enhancement of network activity induced by high-power light stimulation. With simple formulas we calculated the temperature increase due to illumination, taking into account the collected signal, camera properties, and fluorophore efficiency. However, temperature increase calculated in this way is insufficient $\left(<10^{-} 6^{\circ} \mathrm{C}\right)$ to explain the phenomenon of TRPV 4 activation. We also measure the firing rate as a function of the temperature, without retrieving a significant activity enhancement; thus, we can ascribe our effect to a more complex interplay between the channel and exposure to light. The complex pathway leading to the measured effect needs thus further investigation, which is out of the scope of this paper. Possible causes may be linked with the interaction with a different receptor or to a possible sensitivity of the TRPV4 channels to temperature gradients between the internal (absorbing) volume of the cell and the (transparent) recording medium.

\section{DISCUSSION}

Our results are in accordance with previous studies reporting an excitatory effect of visible light exposure on cortical neurons in vivo. In a previous research [25] an fMRI signal was recorded in a rat cortex while exposed to blue laser light stimulation, and another work [26] reports an increasing of prefrontal cortex firing activity in living mice upon laser stimulation at $532 \mathrm{~nm}$. Both studies attributed the activity enhancement to a rising in tissue temperature. It has been previously shown that neurons express TRPVs acting as sensory mediators and activated by endogenous ligands, temperature, and mechanical and osmotic stress [31]. The components of this channel's family contribute to the increase of intracellular calcium by providing or modulating $\mathrm{Ca}^{2+}$ entry pathways and by releasing $\mathrm{Ca}^{2+}$ from intracellular stores [42].

In summary, we investigated the effect of the illumination power on neuronal network activity on in vitro cortical cultures, using a custom-made optical setup. We describe a light-induced increase of synaptic activity mediated by the activation of TRPV4. Our optical setup has been designed to simultaneously acquire and stimulate a wide portion of the sample $\left(2 \times 2 \mathrm{~mm}^{2}\right)$ with a subcellular resolution, allowing the monitoring and stimulation of spontaneous intracellular calcium oscillations at single cell level in a large number of neurons. We demonstrated by optical and patch clamp experiments that high-power LED exposure transiently increased calcium events and spontaneous network activity on cortical cultures, without affecting transient shape (amplitude, rise and decay time) and network synchronization. Moreover, patch clamp experiments made on neurons stimulated by high-power light, in the absence of Fluo4-AM loading, demonstrated that the fluorophore and light together are necessary to increase sEPSC frequency. In conclusion, data obtained from this study suggest that, during LED exposure, the Fluo-4 light absorption caused a release of thermal energy inside the cell and the activation of the thermosensitive channels TRPV4. These results warn those who perform calcium imaging and optogenetics experiments about the possible undesired effect on neuronal activity that should be taken into account. Moreover, even if further studies are necessary to better understand the mechanism behind this phenomenon, our results pave the way to the exploitation of optical stimulation to noninvasively modulate brain signaling, avoiding genetic manipulation.

Funding. Project "Localitis," Fondazione CON IL SUD (Brains2south).

Acknowledgment. Authors acknowledge "Fondazione CON IL" "(Brains2south)," Project "Localitis."

Disclosures. The authors declare no conflicts of interest.

\section{REFERENCES}

1. U. Rossi, "The history of electrical stimulation of the nervous system for the control of pain," Pain Res. Clin. Manage. 15, 5-16 (2003).

2. V. A. Sironi, "Origin and evolution of deep brain stimulation," Front. Integr. Neurosci. 5, 42 (2011).

3. E. Neher, "[6] Correction for liquid junction potentials in patch clamp experiments," in Methods in Enzymology (Elsevier, 1992), Vol. 207, pp. 123-131.

4. O. P. Hamill, A. Marty, E. Neher, B. Sakmann, and F. Sigworth, "Improved patch-clamp techniques for high-resolution current recording from cells and cell-free membrane patches," Pflügers Arch. 391, 85-100 (1981).

5. L. Berdondini, K. Imfeld, A. Maccione, M. Tedesco, S. Neukom, M. Koudelka-Hep, and S. Martinoia, "Active pixel sensor array for high spatio-temporal resolution electrophysiological recordings from single cell to large scale neuronal networks," Lab Chip 9, 2644-2651 (2009).

6. A. C. Thompson, P. R. Stoddart, and E. D. Jansen, "Optical stimulation of neurons," Curr. Mol. Imag. 3, 162-177 (2014). 
7. B. V. Zemelman, G. A. Lee, M. Ng, and G. Miesenböck, "Selective photostimulation of genetically targeted neurons," Neuron 33, 15-22 (2002).

8. B. V. Zemelman, N. Nesnas, G. A. Lee, and G. Miesenböck, "Photochemical gating of heterologous ion channels: remote control over genetically designated populations of neurons," Proc. Natl. Acad. Sci. USA 100, 1352-1357 (2003).

9. S. C. Williams and K. Deisseroth, "Optogenetics," Proc. Natl. Acad. Sci. USA 110, 16287 (2013).

10. J. T. Paz, T. J. Davidson, E. S. Frechette, B. Delord, I. Parada, K. Peng, K. Deisseroth, and J. R. Huguenard, "Closed-loop optogenetic control of thalamus as a tool for interrupting seizures after cortical injury," Nat. Neurosci. 16, 64-70 (2013).

11. R. C. Wykes, J. H. Heeroma, L. Mantoan, K. Zheng, D. C. MacDonald, K. Deisseroth, K. S. Hashemi, M. C. Walker, S. Schorge, and D. M. Kullmann, "Optogenetic and potassium channel gene therapy in a rodent model of focal neocortical epilepsy," Sci. Transl. Med. 4, 161ra152 (2012).

12. V. Gradinaru, F. Zhang, C. Ramakrishnan, J. Mattis, R. Prakash, I. Diester, I. Goshen, K. R. Thompson, and K. Deisseroth, "Molecular and cellular approaches for diversifying and extending optogenetics," Cell 141, 154-165 (2010).

13. A. Berndt, P. Schoenenberger, J. Mattis, K. M. Tye, K. Deisseroth, P. Hegemann, and T. G. Oertner, "High-efficiency channelrhodopsins for fast neuronal stimulation at low light levels," Proc. Natl. Acad. Sci. USA 108, 7595-7600 (2011).

14. S. Satpathy, S. Batabyal, K. R. Dhakal, J. Lin, Y.-T. Kim, and S. K. Mohanty, "Broad spectral excitation of opsin for enhanced stimulation of cells," Opt. Lett. 40, 2465-2468 (2015).

15. W. Deng, E. M. Goldys, M. M. Farnham, and P. M. Pilowsky, "Optogenetics, the intersection between physics and neuroscience: light stimulation of neurons in physiological conditions," Am. J. Physiol. 307, R1292-R1302 (2014).

16. A. Arvanitaki and N. Chalazonitis, Excitatory and Inhibitory Processes Initiated by Light and Infra-Red Radiations in Single Identifiable Nerve Cells (giant ganglion cells of Aplysia) (Pergamon, 1961).

17. R. L. Fork, "Laser stimulation of nerve cells in aplysia," Science $\mathbf{1 7 1}$ 907-908 (1971).

18. J. Wells, C. Kao, K. Mariappan, J. Albea, E. D. Jansen, P. Konrad, and A. Mahadevan-Jansen, "Optical stimulation of neural tissue in vivo," Opt. Lett. 30, 504-506 (2005).

19. M. Chernov and A. W. Roe, "Infrared neural stimulation: a new stimulation tool for central nervous system applications," Neurophotonics 1, 011011 (2014).

20. C.-P. Richter, A. I. Matic, J. D. Wells, E. D. Jansen, and J. T. Walsh, "Neural stimulation with optical radiation," Laser Photon. Rev. 5, 68-80 (2011).

21. M. G. Shapiro, M. F. Priest, P. H. Siegel, and F. Bezanilla, "Thermal mechanisms of millimeter wave stimulation of excitable cells," Biophys. J. 104, 2622-2628 (2013).

22. H. Hirase, V. Nikolenko, J. H. Goldberg, and R. Yuste, "Multiphoton stimulation of neurons," J. Neurobiol. 51, 237-247 (2002).

23. W. D. de Boer, J. J. Hirtz, A. Capretti, T. Gregorkiewicz, M. IzquierdoSerra, S. Han, C. Dupre, Y. Shymkiv, and R. Yuste, "Neuronal photoactivation through second-harmonic near-infrared absorption by gold nanoparticles," Light Sci. Appl. 7, 1-13 (2018).

24. K. Ait Ouares, C. Beurrier, M. Canepari, G. Laverne, and N. Kuczewski, "Opto non-genetics inhibition of neuronal firing," Eur. J. Neurosci. 49, 6-26 (2018).

25. I. N. Christie, J. A. Wells, P. Southern, N. Marina, S. Kasparov, A. V. Gourine, and M. F. Lythgoe, "fMRI response to blue light delivery in the naive brain: implications for combined optogenetic fMRI studies," Neuroimage 66, 634-641 (2013).

26. J. M. Stujenske, T. Spellman, and J. A. Gordon, "Modeling the spatiotemporal dynamics of light and heat propagation for in vivo optogenetics," Cell Rep. 12, 525-534 (2015).

27. S. M. Thompson, L. M. Masukawa, and D. A. Prince, "Temperature dependence of intrinsic membrane properties and synaptic potentials in hippocampal CA1 neurons in vitro," J. Neurosci. 5, 817-824 (1985).

28. J. Kim and B. Connors, "High temperatures alter physiological properties of pyramidal cells and inhibitory interneurons in hippocampus," Front. Cell. Neurosci. 6, 27 (2012).

29. M. Volgushev, T. Vidyasagar, M. Chistiakova, and U. Eysel, "Synaptic transmission in the neocortex during reversible cooling," Neuroscience 98, 9-22 (2000).

30. E. S. Albert, J. M. Bec, G. Desmadryl, K. Chekroud, C. Travo, S. Gaboyard, F. Bardin, I. Marc, M. Dumas, G. Lenaers, C. Hamel, A Muller, and C. Chabbert, "TRPV4 channels mediate the infrared laser-evoked response in sensory neurons," J. Neurophysiol. 107 3227-3234 (2012).

31. A. D. Güler, H. Lee, T. lida, I. Shimizu, M. Tominaga, and M. Caterina, "Heat-evoked activation of the ion channel, TRPV4," J. Neurosci. 22, 6408-6414 (2002).

32. H. Watanabe, J. Vriens, S. H. Suh, C. D. Benham, G. Droogmans, and B. Nilius, "Heat-evoked activation of TRPV4 channels in an HEK293 cell expression system and in native mouse aorta endothelial cells," J. Biol. Chem. 277, 47044-47051 (2002).

33. B. Nilius, J. Vriens, J. Prenen, G. Droogmans, and T. Voets, "TRPV4 calcium entry channel: a paradigm for gating diversity," Am. J. Physiol. 286, C195-C205 (2004).

34. G. M. Beaudoin, III, S.-H. Lee, D. Singh, Y. Yuan, Y.-G. Ng, L. F. Reichardt, and J. Arikkath, "Culturing pyramidal neurons from the early postnatal mouse hippocampus and cortex," Nat. Protocols $\mathbf{7}$, 1741-1754 (2012).

35. H. Bostock, T. Sears, and R. Sherratt, "The effects of 4-aminopyridine and tetraethylammonium ions on normal and demyelinated mammalian nerve fibres," J. Physiol. 313, 301-315 (1981).

36. T. Narahashi, H. G. Haas, and E. F. Therrien, "Saxitoxin and tetrodotoxin: comparison of nerve blocking mechanism," Science 157, 1441-1442 (1967).

37. S. Batabyal, S. Satpathy, L. Bui, Y.-T. Kim, S. Mohanty, R. Bachoo, and D. P. Davé, "Label-free optical detection of action potential in mammalian neurons," Biomed. Opt. Express 8, 3700-3713 (2017).

38. X. Han, X. Qian, J. G. Bernstein, H.-H. Zhou, G. T. Franzesi, P. Stern, R. T. Bronson, A. M. Graybiel, R. Desimone, and E. S. Boyden, "Millisecond-timescale optical control of neural dynamics in the nonhuman primate brain," Neuron 62, 191-198 (2009).

39. G. Palazzolo, M. Moroni, A. Soloperto, G. Aletti, G. Naldi, M. Vassalli, T. Nieus, and F. Difato, "Fast wide-volume functional imaging of engineered in vitro brain tissues," Sci. Rep. 7, 8499 (2017).

40. B. F. Grewe, D. Langer, H. Kasper, B. M. Kampa, and F. Helmchen, "High-speed in vivo calcium imaging reveals neuronal network activity with near-millisecond precision," Nat. Methods 7, 399-405 (2010).

41. F. Vincent, A. Acevedo, M. T. Nguyen, M. Dourado, J. DeFalco, A. Gustafson, P. Spiro, D. E. Emerling, M. G. Kelly, and M. A. Duncton, "Identification and characterization of novel TRPV4 modulators," Biochem. Biophys. Res. Commun. 389, 490-494 (2009).

42. S. F. Pedersen and B. Nilius, "Transient receptor potential channels in mechanosensing and cell volume regulation," in Methods in Enzymology (Elsevier, 2007), Vol. 428, pp. 183-207. 


\section{Queries}

1. AU: Please check the edits made to the title of this paper.

2. AU: You have indicated that Figs. 1,2,3,5,6,7 are to be printed in color. Please confirm this to ensure that your order is reflected in the print issue.

3. AU: OSA policy requires many acronyms to be defined. In the sentence beginning "We investigated the possible origin of the phenomena... " please check my definition of "TRPV4."

4. AU: In the sentence beginning "Earlier studies identified one of the potential mechanisms..." please check my definition of "TRPV4."

5. AU: Please check the hierarchy of section level heading.

6. AU: In the sentence beginning "We synchronized the LED light and camera recording...," please check my edit (to confirm that "Data "AcQuisition" as you presented it is not the name of a company of specific product.

7. AU: The funding information for this article has been generated using the information you provided to OSA at the time of article submission. Please check it carefully. If any information needs to be corrected or added, please provide the full name of the funding organization/institution as provided in the CrossRefOpen Funder Registry (https://search.crossref.org/funding). 